\title{
Interferências dos pedoambientes nos atributos do solo em uma topossequência de transição Campos/Floresta ${ }^{1}$
}

\author{
Interference of the pedoenvironment on soil properties in a topossenquence of the \\ Grassland/Forest transition
}

\author{
Milton César Costa Campos ${ }^{2 *}$, Mateus Rosas Ribeiro ${ }^{3}$, Valdomiro Severino de Souza Júnior ${ }^{3}$, Mateus Rosas \\ Ribeiro Filho $^{3}$ e Ivanildo Amorim de Oliveira ${ }^{4}$
}

\begin{abstract}
Resumo - As condições pedoambientais promovem alterações nos atributos do solo. Sendo assim, o objetivo deste trabalho foi estudar as interferências dos pedoambientes nos atributos do solo de uma topossequência de transição Campos/Floresta na região de Humaitá, AM. Escolheu-se uma topossequência característica de sequência Campos/ Floresta, em seguida foi estabelecido um caminhamento, partindo do ambiente de campo natural até o ambiente de floresta. Esses pedoambientes foram identificados e delimitados conforme a expressão dos padrões vegetacionais. Foram coletadas 20 amostras laterais ao caminhamento, representativas de cada uma das unidades pedoambientais da topossequência (campo alto: 0,0-14 e 0,30-0,66 m; campo baixo: 0,0-0,15 e 0,27-0,80 m; zona de ecótono: 0,0-0,15 e $0,32-0,50 \mathrm{~m}$; floresta: $0,0-0,15$ e $0,67-100 \mathrm{~m}$ ), totalizando 80 amostras. O critério de escolha das profundidades foi a coincidência com os horizontes diagnósticos superficiais e subsuperficiais determinados na descrição morfológica dos perfis. Foram realizadas análises físicas de textura, argila dispersa e grau de floculação, densidades do solo e das partículas, porosidade total e condutividade hidráulica. Nas análises químicas foram determinados $\mathrm{pH}$ em água e $\mathrm{KCl}$, $\mathrm{Ca}, \mathrm{Mg}, \mathrm{K}, \mathrm{Na}$ e $\mathrm{Al}$ trocáveis, $\mathrm{P}$ disponível, $\mathrm{H}+\mathrm{Al}$ e C orgânico. Os atributos físicos e químicos do solo apresentaram-se dependentes dos pedoambientes. Com o uso das técnicas estatísticas multivariadas foi possível distinguir três diferentes ambientes que equivalem a três pedoambientes.
\end{abstract}

Palavras-chave - Solos da Amazônia. Campos Naturais. Pedogênese.

\begin{abstract}
The pedoenvironmental conditions promote changes in soil properties, so the aim of this work was to study Interference of the pedoenvironment on soil properties in a topossequence of the Grassland/Forest transition in the Humaitá region, AM. Was picked up a topossequence characteristic sequence Grassland/Forest, then a pathway was established, from a field environment to the natural forest environment. These pedoambients were identified and defined as the expression patterns of vegetation. Were collected 20 samples at the representative side of the profiles in each one of the pedoambiental units of the topossequence (top grassland: $0.0-14$ and $0.30-0.66 \mathrm{~m}$;low grassland : 0.0-0.15 and 0.27-0.80 m; ecotone zone: $0.0-0.15$ and 0.32-0.50 m; forest: $0.0-0.15 \mathrm{~m} 0.67-100$ as a total of 80 samples) being the criteria for sampling depths the coincidence with the surface and subsurface diagnostic horizons determined by the morphological description of the profiles. Were performed physical analysis of texture, clay dispersion and flocculation, soil bulk density and particle porosity and hydraulic conductivity. In the chemical analysis were determined $\mathrm{pH}$ in water and $\mathrm{KCl}, \mathrm{Ca}, \mathrm{Mg}, \mathrm{K}, \mathrm{Na}$ and $\mathrm{Al}$ contents, available $\mathrm{P}, \mathrm{H}+\mathrm{Al}$ and organic carbon. The physical and chemical soil were dependent on the pedoambients. With the use of multivariate statistical techniques was possible to distinguish three different environments that are equivalent to three pedoambients.
\end{abstract}

Key words - Soils of Amazon. Native Grassland. Pedogenesis.

\footnotetext{
* Autor para correspondência

${ }^{1}$ Recebido para publicação em 23/03/2010; aprovado em 13/11/2010

Parte da Tese do primeiro autor apresentada no Programa de Pós-Graduação em Ciências do Solo da UFRPE

${ }^{2}$ Colegiado de Agronomia, IEAA/UFAM, Humaitá-AM, Brasil, mcesarsolos@gmail.com

${ }_{3}^{3}$ Departamento de Agronomia, UFRPE, Recife-PE, Brasil, mrosas@depa.ufrpe.br, vsouzajr@yahoo.com, mateusrf@depa.ufrpe.br

${ }^{4}$ Colegiado de Agronomia, IEAA/UFAM, Humaitá-AM, Brasil, ivanildoufam@gmail.com
} 


\section{Introdução}

A região Sul do Amazonas apresenta várias fitofisionomias. Uma destas é denominada de Campos Naturais, que compreende as áreas dos "Campos de Puciari - Humaitá", que inclui várias formações campestres, onde se alternam, às vezes, pequenas árvores isoladas e galerias florestais ao longo dos igarapés (BRAUN; RAMOS, 1959). De acordo com Freitas et al. (2002), esses campos formam alguns mosaicos com as florestas circundantes, sendo o contato entre essas vegetações, em alguns locais, de maneira abrupta, mas em outros, a mudança da vegetação entre a floresta e o campo natural é gradual.

Segundo Campos (2009), a expressão da vegetação é reflexo das condições topográficas e do solo, pois à medida que o ocorre elevação do terreno, vai mudando de fisionomia, cedendo lugar a uma formação de aspecto mais uniforme, dotada de árvores mais baixas, que constitui o "cerrado". Esta formação por sua vez transforma-se, também gradativamente, com espaçamento cada vez maior entre as árvores e o aparecimento de vegetação rasteira, que domina totalmente logo depois, constituindo os campos propriamente ditos (MARTINS et al., 2006a).

Referindo-se às interfaces solo-vegetação, Yimer et al. (2006) destacam que a diversidade fitofisionômica relaciona-se a variação de características edáficas, especialmente a disponibilidade de nutrientes e o teor de alumínio no solo. Por outro lado, Rezaei e Gilkes (2005) sugerem que há uma estreita relação entre fitofisionomias e a variação das formas de relevo e topografia, bem como suas interferências na dinâmica da água no solo. Nesse sentido, Franzen et al. (2006) afirmam que as condições pedoambientais promovem alterações nos atributos do solo, sendo estas dependentes de características, como forma do relevo, quantidade e fluxos de água e características dos materiais originários, o que vem refletir na expressão da vegetação nativa.

Além dos aspectos físicos do ambiente, também são importantes as ferramentas estatísticas que validem de maneira satisfatória as demandas do estudo. Nesse sentido, Hair et al. (2005) destacam as técnicas estatísticas multivariadas, pois se fundamentam na utilização do sistema de classificação numérica e hierárquica, onde a variabilidade é minimizada dentro de um grupo e maximizada entre os grupos, produzindo grupos relativamente homogêneos.

Vários estudos têm aplicado com sucesso, nas técnicas multivariadas, as investigações de variáveis do solo. De acordo com Sena et al. (2002), uma das vantagens destas técnicas é a formação de agrupamento de populações com características similares, permitindo um melhor entendimento das variações dos processos que ocorrem no solo. Para Fu et al. (2004), com o uso destas técnicas consegui-se estabelecer inter-relações entre os aspectos topográficos e os atributos do solo. Yemefack et al. (2005), por sua vez, destacam que este instrumento estatístico permite agrupar dados relacionados aos atributos do solo, favorecendo a elucidação dos processos responsáveis pela variabilidade espacial do solo.

Portanto, o conhecimento das condições dos pedoambientes permite entender o funcionamento dos ecossistemas, além de ser uma premissa básica para estabelecimento de práticas adequadas de manejo do solo. Sendo assim, o objetivo deste trabalho foi estudar as interferências dos pedoambientes nos atributos do solo de uma topossequência de transição Campos/Floresta na região de Humaitá, AM.

\section{Material e métodos}

\section{Localização e caracterização ambiental da área estudada}

A área de estudo localiza-se na região de Humaitá, sul do Estado do Amazonas, estando situada sob as coordenadas geográficas de 7³0'24" S e 6304'56" W, com altitude média de 25 metros (FIG. 1). A região apresenta relevo aproximado ao do tipo "tabuleiro", com desníveis muito pequenos e bordos ligeiramente abaulados. Essas terras mais altas constituem os divisores topográficos de água entre os rios da região. O desnível dessas zonas mais elevadas e os vale dos igarapés, é da ordem de 15 a 29 metros, ocorrendo, entretanto, de maneira súbita (BRAUN; RAMOS, 1959).

Com relação à geologia, a toposseqüência estudada foi localizada sobre uma área formada a partir de sedimentos aluviais indiferenciados ou antigos, que são cronologicamente oriundos do Holoceno. Os sedimentos desta formação são advindos de dois ciclos de sedimentação: a) bancos arenosos, inferiores, que representam a sedimentação plúviofluvial e b) sedimentos argilosos superiores, indicando sedimentação lacustrina. A região apresenta uma vegetação de contato entre Campos e Florestas que se caracteriza por áreas que inclui varias formações campestres onde a vegetação que prevalece é a gramíneo-lenhosa baixa e se alterna com pequenas árvores isoladas e galerias florestais ao longo dos rios (BRAUN; RAMOS, 1959). 


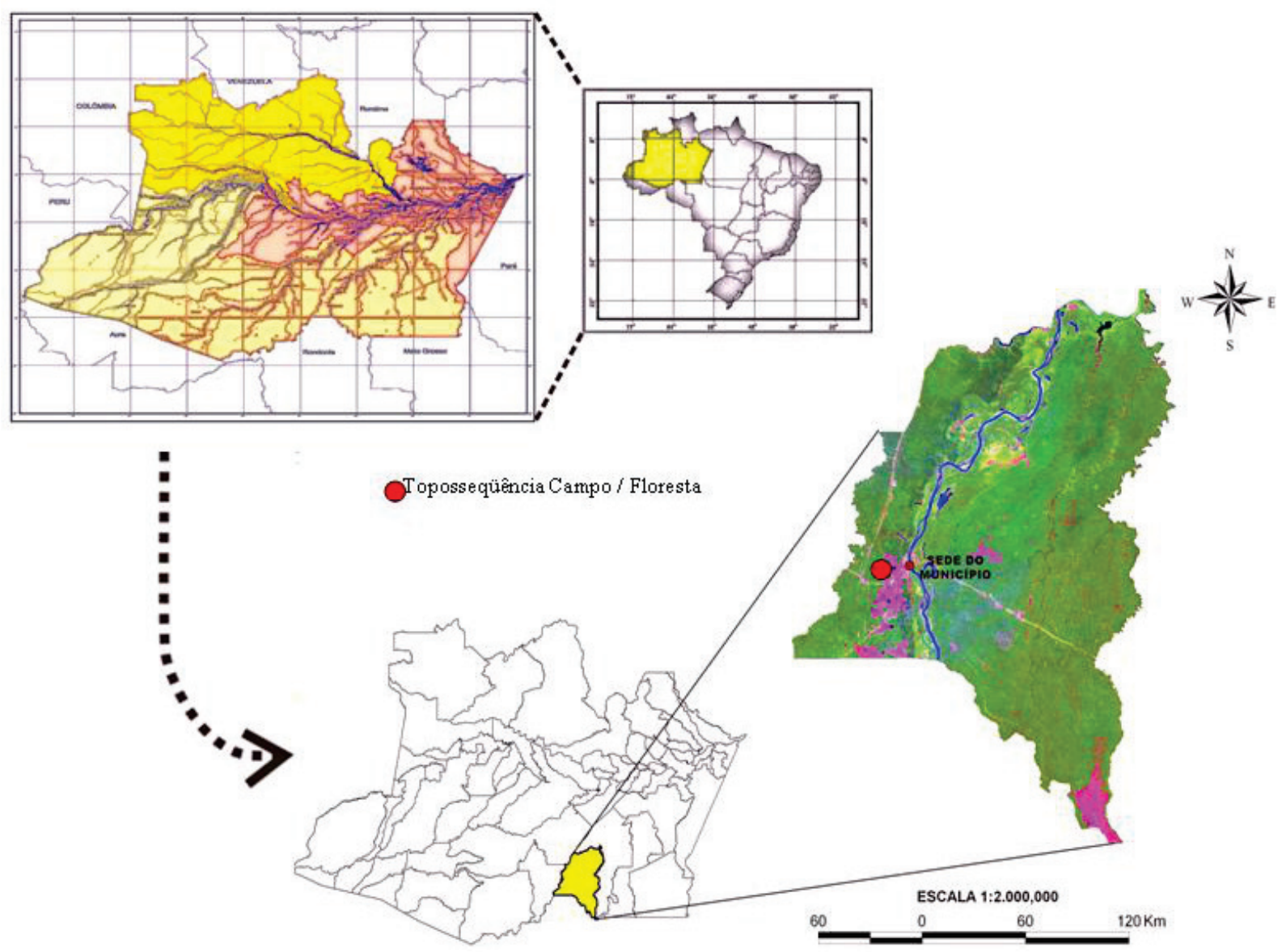

Figura 1 - Mapa de localização da toposseqüência de transição Campos/Floresta na região de Humaitá, AM

O clima da região, segundo a classificação de Köppen, é do tipo tropical chuvoso (chuvas do tipo monção), apresentando um período seco de pequena duração (Am), temperaturas variando entre 25 e $27{ }^{\circ} \mathrm{C}$ e precipitação média anual de $2.500 \mathrm{~mm}$, com período chuvoso iniciando em outubro e prolongando-se até junho e umidade relativa do ar entre 85 e $90 \%$.

\section{Métodos de campo, laboratório e escritório}

Utilizando-se do critério da repetição (paisagem que mais ocorre na região) e da preservação dos ambientes (ambiente não antropizado), escolheu-se uma área com sequência Campos/Floresta (FIG. 2). Em seguida foi estabelecido um caminhamento, partido do ambiente de campo natural até o ambiente de floresta. A transeção cortou os diferentes pedoambientes existentes. Esses pedoambientes foram identificados e delimitados conforme a expressão dos padrões vegetacionais e pedológicos.

Foram abertas trincheiras nos pedoambientes da topossequência mapeada para caracterização morfológica, física, química e mineralógica. A identificação dos horizontes e a descrição morfológica foram realizadas conforme Santos et al. (2005), com coleta de amostras dos horizontes dos solos. Os solos foram classificados segundo critérios estabelecidos por SiBCS (EMBRAPA, 2006).

Foram coletadas 20 amostras laterais aos perfis representativos das diversas unidades pedoambientais da topossequência (campo alto: 0,0-14 e 0,30-0,66 m; campo baixo: $0,0-0,15$ e $0,27-0,80 \mathrm{~m}$; zona de ecótono: $0,0-0,15$ e 0,32- 0,50 m; floresta: 0,0-0,15 e 0,67-100 m totalizando 80 amostras) e o critério de escolha das profundidades foi a coincidência com os horizontes diagnósticos superficiais e subsuperficiais.

A análise granulométrica foi realizada pelo método da pipeta, utilizando uma solução de $\mathrm{NaOH}$ como dispersante químico, e agitação mecânica por 10 minutos. A fração argila foi separada por sedimentação, as areias grossa e fina por tamisação e o silte calculado por diferença. Foi determinada a argila dispersa em água e feito o cálculo do grau de floculação, segundo metodologia da Embrapa (1997). 


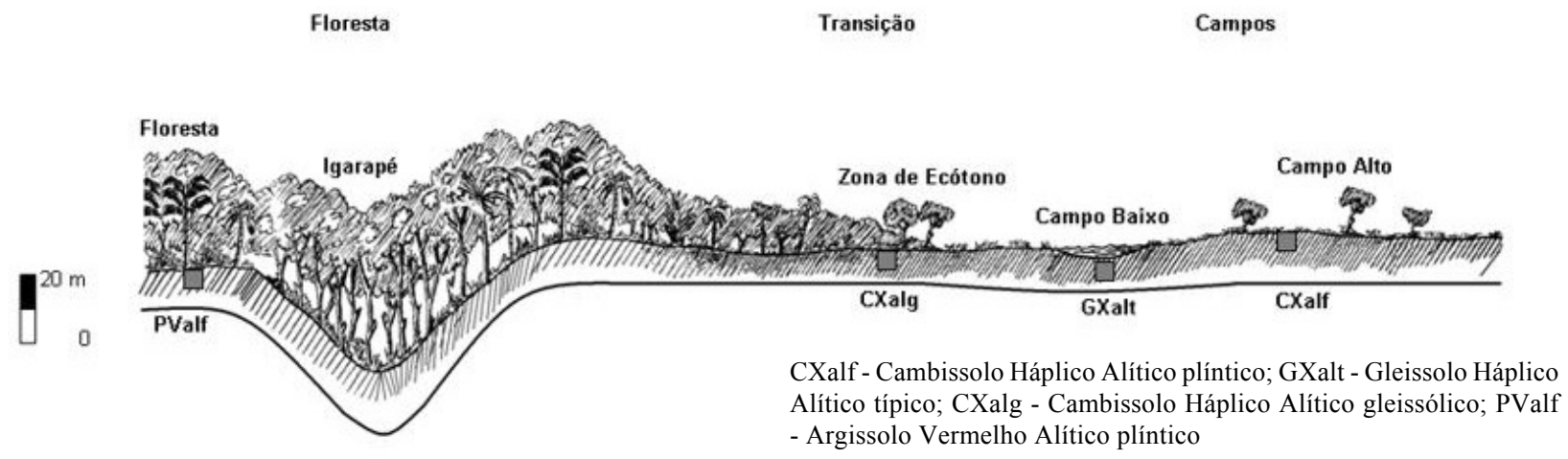

Figura 2 - Perfil esquemático mostrando a vegetação, relevo e as classes de solos em toposseqüência típica da transição Campos/ Floresta na região de Humaitá, AM

Fonte: Adaptado de Braun \& Ramos (1959)

A densidade do solo foi obtida pelo método do anel volumétrico e a densidade de partícula pelo método do balão volumétrico, conforme metodologia da Embrapa (1997). A porosidade total foi calculada a partir dos dados obtidos das densidades do solo e de partícula, empregandose a seguinte expressão:

$$
P t=100\left(1-\frac{D s}{D p}\right)
$$

$\mathrm{Pt}=$ porosidade total; $\mathrm{Ds}=$ densidade do solo; $\mathrm{Dp}=$ densidade de partícula

A condutividade hidráulica saturada dos solos foi determinada em amostras deformadas (TFSA), conforme metodologia proposta pela Embrapa (1997)

Cálcio, magnésio e alumínio trocáveis foram extraídos com $\mathrm{KCl} 1 \mathrm{~mol} \mathrm{~L}^{-1}$; potássio, sódio e fósforo disponível, foram extraídos com solução de Mehlich-1; e a acidez potencial $(\mathrm{H}+\mathrm{Al})$ foi extraída com solução tamponada a pH 7,0 de acetato de cálcio, utilizando-se metodologia proposta pela Embrapa (1997). Com base nos resultados das análises químicas, foram calculadas a soma de bases (SB), a capacidade de troca catiônica (CTC), a saturação por bases (V\%) e alumínio.

$\mathrm{O} \mathrm{pH}$ foi determinado potenciometricamente, utilizando-se relação 1:2,5 de solo: em água e $\mathrm{KCl}$ (EMBRAPA, 1997).

O carbono orgânico total foi determinado pelo método de oxidação via úmida, com aquecimento externo (YEOMANS; BREMNER, 1988), e a matéria orgânica foi estimada com base no carbono orgânico total.

Com os resultados das amostras coletadas nos ambientes estudados, foram realizadas análises de variância univariada e os resultados foram comparados pelo teste de Tukey a $5 \%$ de probabilidade, sendo utilizado o programa estatístico Statitica 7.0 (STATITICA, 2005).

Com o objetivo de avaliar a ação conjunta dos atributos físicos (areia fina, grossa, silte, argila, densidade do solo e partícula, porosidade total, e condutividade hidráulica do solo saturado) e químicos $(\mathrm{pH}$ em água e $\mathrm{KCl}, \mathrm{P}, \mathrm{C}, \mathrm{Ca}^{2+}, \mathrm{Mg}^{2+}, \mathrm{K}^{+}, \mathrm{Na}^{+}, \mathrm{Al}^{3+}$ e $\mathrm{H}^{+}+\mathrm{Al}^{3+}$ ) na discriminação dos ambientes estudados,foram aplicadas às amostras de solos dos pedoambientes, dois métodos estatísticos multivariados, visando classificar os acessos em grupos: análise hierárquica de agrupamentos e análise de componentes principais. Todas as análises multivariadas foram realizadas após a padronização das variáveis em que cada uma ficou com média 0 e variância 1 . A análise hierárquica de agrupamentos foi realizada calculando-se a distância euclidiana entre os acessos, para o conjunto das vinte e duas variáveis, e utilizando o algoritmo de Ward para a obtenção dos agrupamentos de acessos similares. O resultado da análise foi apresentado em forma gráfica (dendrograma) que auxiliou na identificação dos agrupamentos dos acessos.

A análise de componentes principais permite condensar a maior quantidade da informação original contida em $\mathrm{p}$ variáveis $(\mathrm{p}=22$, neste estudo) em duas variáveis latentes ortogonais denominadas componentes principais, que são combinações lineares das variáveis originais criadas com os dois maiores autovalores da matriz de covariância dos dados (HAIR, 2005). Desta forma, o conjunto inicial de vinte duas variáveis passou a ser caracterizado por duas novas variáveis latentes, o que possibilitou sua localização em figuras bidimensionais (ordenação dos acessos por componentes principais). A adequação desta análise é verificada pela quantidade da informação total das 
variáveis originais retida pelos componentes principais que mostram autovalores superiores à unidade (KAISER, 1958). Autovalores inferiores à unidade não dispõem de informação relevante.

Todas as análises estatísticas foram processadas com auxilio do programa estatístico Statistica 7.0 (STATITICA, 2005).

\section{Resultados e discussão}

Na Tabela 1 são apresentados os resultados referentes aos atributos físicos. Em relação às frações granulométricas, o silte foi a fração dominante em todos os pedoambientes, fato justificável pela natureza aluvial dos sedimentos que constituem o material de origem (BRASIL, 1978). Comparando os diversos pedoambientes, observa-se que no campo baixo e na zona de ecótono os teores de silte são mais elevados, diferindo estatisticamente dos ambientes de campo alto e floresta. Segundo Rosolen e Herpin, (2008) esse comportamento se deve, principalmente, à posição rebaixada, e ocorrência em depressões topográficas que favorecem a deposição de sedimentos mais finos.

A fração areia nos dois horizontes diagnósticos apresentou-se mais elevadas nas áreas de campo alto e floresta, diferindo estatisticamente dos demais pedoambientes. A fração argila apresentou o seguinte comportamento: nos horizontes diagnósticos superficiais exibiu maiores teores no pedoambiente de campo baixo, seguido pela zona de ecótono, campo alto e floresta, para os horizontes diagnósticos subsuperficiais verificou-se que o campo alto e a zona de ecótono apresentaram os maiores valores, diferindo dos demais pedoambientes estudados. Este fato possivelmente esteja relacionado à formação lacustrina do ambiente, conforme Carvalho (1986), que trabalhou com solos da região de Humaitá, AM.

A densidade do solo apresentou pequenas variações, com os maiores valores nos horizontes superficiais dos pedoambientes de campo alto e floresta quando comparados aos pedoambientes de zona de ecótono e campo baixo. Já para os horizontes subsuperficiais observou-se que o campo alto, floresta e zona de ecótono diferem estatisticamente do campo baixo (TAB. 1). A densidade de partícula por sua vez, mostrou-se semelhantemente em todos os horizontes dos pedoambientes estudados, evidenciando materiais minerais de mesma natureza, corroborando resultados encontrados por Martins (2001).

A porosidade total apresentou diferenças significativas apenas para os horizontes diagnósticos superficiais, exibindo valores mais elevados nas áreas de campo baixo e zona de ecótono (TAB. 1), coincidindo nos ambientes com maiores conteúdos de matéria orgânica, corroborando assim com os resultados encontrados por Martins et al. (2006a) na região sul do Amazonas.

O resultado estatístico dos atributos químicos é apresentado na Tabela 2. Os valores do $\mathrm{pH}$ em água, em superfície, não apresentaram diferenças estatísticas entre os ambientes. Em subsuperfície constatou-se que o campo baixo apresentou valores mais altos e diferenças estatísticas em relação aos demais pedoambientes. Em relação ao $\mathrm{pH}$ em $\mathrm{KCl}$, nota-se que o solo do

Tabela 1 - Médias dos atributos físicos nos diversos pedoambientes em uma sequência de transição Campos/Floresta na região de Humaitá, AM

\begin{tabular}{|c|c|c|c|c|c|c|}
\hline \multirow{2}{*}{ Pedoambientes } & Areia & Silte & Argila & Ds & $\mathrm{Dp}$ & $\mathrm{Pt}$ \\
\hline & & $\mathrm{g} \mathrm{kg}^{-1}$ & & \multicolumn{2}{|c|}{$\mathrm{g} \mathrm{cm}^{-3}$} & $\%$ \\
\hline \multicolumn{7}{|c|}{ Horizontes Diagnósticos Superficiais } \\
\hline Campo Alto & $337,3 \mathrm{a}$ & $460,3 \mathrm{c}$ & $202,4 \mathrm{c}$ & $1,22 \mathrm{a}$ & $2,61 \mathrm{a}$ & $52 \mathrm{~b}$ \\
\hline Campo Baixo & $49,3 \mathrm{~b}$ & $621,7 \mathrm{~b}$ & $329,0 \mathrm{a}$ & $0,99 \mathrm{c}$ & $2,50 \mathrm{a}$ & $60 \mathrm{a}$ \\
\hline Zona de Ecótono & $79,7 \mathrm{~b}$ & $663,6 \mathrm{a}$ & $256,7 \mathrm{~b}$ & $1,08 \mathrm{~b}$ & $2,57 \mathrm{a}$ & $58 \mathrm{a}$ \\
\hline Floresta & $402,0 \mathrm{a}$ & $407,7 \mathrm{~d}$ & $189,3 \mathrm{c}$ & $1,21 \mathrm{a}$ & $2,64 \mathrm{a}$ & $54 \mathrm{~b}$ \\
\hline \multicolumn{7}{|c|}{ Horizontes Diagnósticos Subsuperficiais } \\
\hline Campo Alto & $255,29 \mathrm{~b}$ & $426,80 \mathrm{~b}$ & $317,90 \mathrm{a}$ & $1,32 \mathrm{a}$ & $2,72 \mathrm{a}$ & $51 \mathrm{a}$ \\
\hline Campo Baixo & $118,11 \mathrm{c}$ & $630,93 \mathrm{a}$ & $250,96 \mathrm{~b}$ & $1,27 \mathrm{~b}$ & $2,66 \mathrm{a}$ & $52 \mathrm{a}$ \\
\hline Zona de Ecótono & $70,58 \mathrm{~d}$ & $607,65 \mathrm{a}$ & $321,76 \mathrm{a}$ & $1,32 \mathrm{a}$ & $2,74 \mathrm{a}$ & $51 \mathrm{a}$ \\
\hline Floresta & 339,26 a & $415,20 \mathrm{~b}$ & $245,54 \mathrm{~b}$ & $1,32 \mathrm{a}$ & $2,69 \mathrm{a}$ & $51 \mathrm{a}$ \\
\hline
\end{tabular}

Campo Alto $=20$ pontos $;$ Campo Baixo $=20$ pontos; Zona de Ecótono $=20$ pontos $;$ Floresta $=20$ pontos, totalizando 80 pontos. Ds $=$ densidade do solo; $\mathrm{Dp}=$ densidade de partícula; $\mathrm{Pt}=$ porosidade total. Médias seguidas de mesmas letras nas colunas não diferem entre si pelo teste de Tukey ao nível de $5 \%$ 
Tabela 2 - Médias dos atributos químicos nos diversos pedoambientes em uma seqüência de transição Campos/Floresta na região de Humaitá, AM

\begin{tabular}{|c|c|c|c|c|c|c|c|c|c|c|c|c|}
\hline \multirow{2}{*}{${ }^{1} \mathrm{PA}$} & \multicolumn{2}{|c|}{$\mathrm{pH}$} & \multirow{2}{*}{$\begin{array}{c}\mathrm{MO} \\
\mathrm{g} \mathrm{kg}^{-1}\end{array}$} & \multirow{2}{*}{$\frac{P}{\mathrm{mg} \mathrm{kg}^{-1}}$} & \multirow{2}{*}{$\mathrm{Ca}$} & \multirow[t]{2}{*}{$\mathrm{Mg}$} & \multirow[t]{2}{*}{$\mathrm{K}$} & \multirow{2}{*}{$\begin{array}{r}\mathrm{Al} \\
\mathrm{kg}_{\mathrm{c}} \mathrm{kg}^{-1}\end{array}$} & \multirow[t]{2}{*}{ SB } & \multirow{2}{*}{ CTC } & \multirow[t]{2}{*}{$\mathrm{V}$} & \multirow[t]{2}{*}{$\mathrm{m}$} \\
\hline & $\mathrm{H}_{2} \mathrm{O}$ & $\mathrm{KCl}$ & & & & & & & & & & \\
\hline \multicolumn{13}{|c|}{ Horizontes Diagnósticos Superficiais } \\
\hline C. Alto & $4,3 \mathrm{a}$ & $3,8 \mathrm{~b}$ & $1,9 \mathrm{~b}$ & $0,8 \mathrm{~b}$ & $0,4 \mathrm{a}$ & $0,2 \mathrm{a}$ & $0,06 \mathrm{~b}$ & $3,0 \mathrm{c}$ & $0,7 \mathrm{a}$ & $4,9 \mathrm{a}$ & $34,8 \mathrm{a}$ & $81,4 \mathrm{~b}$ \\
\hline C.Baixo & $4,4 \mathrm{a}$ & $3,9 \mathrm{a}$ & $3,0 \mathrm{a}$ & $1,1 \mathrm{a}$ & $0,4 \mathrm{a}$ & $0,2 \mathrm{a}$ & $0,04 \mathrm{~b}$ & $2,7 \mathrm{c}$ & $0,7 \mathrm{a}$ & $11,4 \mathrm{a}$ & $6,2 \mathrm{~b}$ & $80,4 \mathrm{~b}$ \\
\hline Z.Ecótono & $4,3 \mathrm{a}$ & $3,5 \mathrm{~d}$ & $2,0 \mathrm{~b}$ & $1,0 \mathrm{a}$ & $0,3 \mathrm{ab}$ & $0,1 \mathrm{a}$ & $0,11 \mathrm{a}$ & $5,3 \mathrm{a}$ & $0,6 \mathrm{a}$ & $9,3 \mathrm{a}$ & $10 \mathrm{ab}$ & $90,3 \mathrm{a}$ \\
\hline Floresta & $4,0 \mathrm{a}$ & $3,6 \mathrm{c}$ & $1,3 \mathrm{c}$ & $0,6 \mathrm{~b}$ & $0,2 \mathrm{~b}$ & $0,2 \mathrm{a}$ & $0,09 \mathrm{a}$ & $3,8 \mathrm{~b}$ & $0,6 \mathrm{a}$ & $9,3 \mathrm{a}$ & $7,9 \mathrm{ab}$ & $85,8 \mathrm{ab}$ \\
\hline \multicolumn{13}{|c|}{ Horizontes Diagnósticos Subsuperficiais } \\
\hline C. Alto & $4,5 \mathrm{~b}$ & $3,7 \mathrm{a}$ & $0,8 \mathrm{a}$ & $1,2 \mathrm{a}$ & $0,4 \mathrm{a}$ & $0,2 \mathrm{ab}$ & $0,04 \mathrm{~b}$ & $5,2 \mathrm{ab}$ & $0,8 \mathrm{ab}$ & $4,2 \mathrm{~b}$ & $29,2 \mathrm{a}$ & $86,7 \mathrm{bc}$ \\
\hline C. Baixo & $4,9 \mathrm{a}$ & $3,6 \mathrm{~b}$ & $0,7 \mathrm{a}$ & $1,2 \mathrm{a}$ & $0,3 \mathrm{~b}$ & $0,4 \mathrm{a}$ & $0,04 \mathrm{~b}$ & $5,0 \mathrm{ab}$ & $0,9 \mathrm{a}$ & $6,9 \mathrm{ab}$ & $18 \mathrm{ab}$ & $83,7 \mathrm{c}$ \\
\hline Z.Ecótono & $4,4 \mathrm{~b}$ & $3,6 \mathrm{~b}$ & $0,8 \mathrm{a}$ & $1,0 \mathrm{a}$ & $0,3 \mathrm{c}$ & $0,2 \mathrm{~b}$ & $0,06 \mathrm{a}$ & $6,2 \mathrm{a}$ & $0,6 \mathrm{~b}$ & $10,9 \mathrm{a}$ & $6,0 \mathrm{~b}$ & $90,5 \mathrm{a}$ \\
\hline Floresta & $4,4 \mathrm{~b}$ & $3,7 \mathrm{a}$ & $0,7 \mathrm{a}$ & $0,7 \mathrm{~b}$ & $0,3 \mathrm{c}$ & $0,3 \mathrm{ab}$ & $0,05 \mathrm{~b}$ & $4,7 \mathrm{~b}$ & $0,6 \mathrm{~b}$ & $10,4 \mathrm{a}$ & $6,5 \mathrm{~b}$ & $87,6 \mathrm{ab}$ \\
\hline
\end{tabular}

${ }^{1}$ Pedoambientes; $\mathrm{C}$. Alto $=20$ pontos; C. Baixo $=20$ pontos; Z. de Ecótono $=20$ pontos; Floresta $=20$ pontos, totalizando 80 pontos. Médias seguidas de mesmas letras nas colunas não diferem entre si pelo teste de tukey ao nível de $5 \%$

campo baixo apresentou os maiores resultados. Já em subsuperfície os pedoambientes de floresta e campo alto apresentaram os valores mais elevados em relação ao campo baixo e zona de ecótono.

Os teores de matéria orgânica nos horizontes superficiais foram maiores no solo do campo baixo, diferindo estatisticamente dos demais pedoambientes, não havendo diferenças estatísticas entre os horizontes subsuperficiais (TAB. 2). Estes resultados são atribuídos ao maior acúmulo de material orgânicos associado à má drenagem deste ambiente, diminuído a decomposição (MARTINS et al., 2006a). Para os teores de P disponível nos horizontes diagnósticos superficiais, os maiores teores foram observados nos pedoambientes de campo baixo e zona de ecótono. Segundo Melo et al. (2006) estes resultados são influenciados pela topografia que favorece o carreamento e acúmulo de nutrientes.

Os teores de $\mathrm{Ca}^{2+}, \mathrm{Mg}^{2+}$ e $\mathrm{K}^{+}$trocáveis, apesar de muito baixos em todos os pedoambientes estudados, apresentaram algumas diferenças entre si, podendo-se destacar, por exemplo, que em superfície, o do $\mathrm{Ca}^{2+}$ foi mais baixo no pedoambiente de floresta, enquanto em subsuperfície, foi significativamente mais alto no campo alto. $\mathrm{O} \mathrm{Mg}^{2+}$, por sua vez, não apresentou diferenças estatísticas em superfície, o quê, de acordo com Franzen et al. (2006), deve-se possivelmente às pequenas variações do relevo ou ao material de origem.

De maneira geral os valores da acidez trocável $\left(\mathrm{Al}^{3+}\right)$ foram elevados, apresentando na superfície valores menores que em subsuperfície devido aos maiores teores de matéria orgânica nesta, conforme destaca Martins et al. (2006b).
Baseando-se no coeficiente de aglomeração, foi admitido um corte na distância de ligação de 5,0 para os horizontes diagnóstico superficiais, favorecendo a formação de três grupos com características distintas: G1 referente à zona de ecótono, G2 ao campo baixo e G3 contemplando os pedoambientes de floresta e campo alto que, neste caso, provavelmente apresentaram atributos físicos e químicos semelhantes haja vista a formação um único agrupamento (FIG. 3). Esta informação corrobora com a afirmativa de Yemefack et al. (2005) de que esta técnica permite agrupar variáveis com características semelhantes entre si e com aumento de variabilidade entre os agrupamentos formados.

Utilizando-se também do coeficiente de aglomeração para os horizontes diagnósticos subsuperficiais, considerando-se o corte a uma distância de ligação de 5,0, foi verificada a formação nítida de três grupos (G1, G2 e G3) distintos, G1 correspondendo ao campo baixo, G2 contemplando a zona de ecótono e floresta e G3 englobando o campo alto (FIG. 3). Fu et al. (2004), estudando as relações entre os atributos do solo, topografia e vegetação em Beijing na China, destaca a análise de agrupamento como uma ferramenta bastante eficiente no estudo de múltiplos fatores ambientais.

Segundo Boruvka e Kozak, (2001), as análises de distribuição espacial que consideram os diferentes solos nas paisagens com o uso da estatística multivariada podem contribuir para a obtenção de um melhor entendimento do ambiente. As técnicas de estatística multivariada levam em consideração a totalidade das 
variáveis pois uma depende da outra e as informações são fornecidas pelo conjunto e não individualmente (FREDDI et al., 2008).
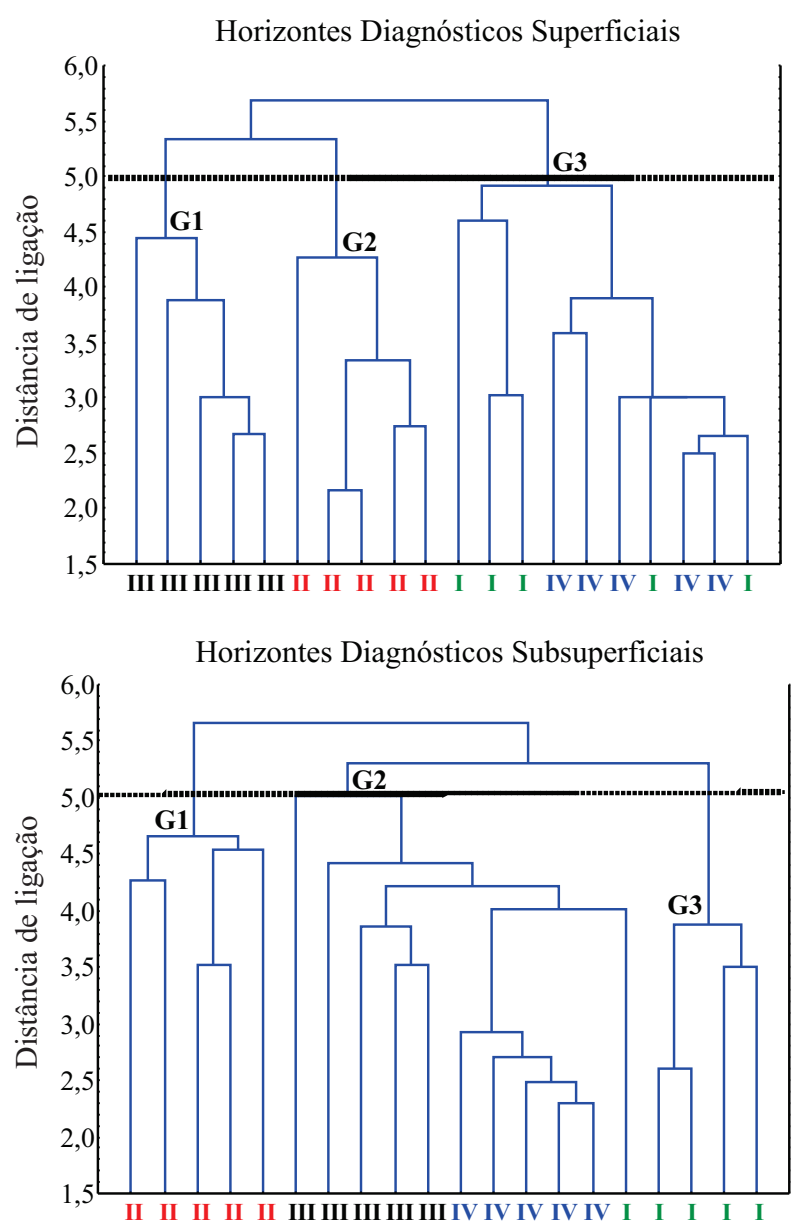

$\mathbf{I}=$ Campo Alto; $\mathbf{I I}=$ Campo Baixo; $\mathbf{I I I}=$ Zona de Ecotóno; $\mathbf{I V}=$ Floresta

Figura 3 - Dendrograma das interrrelações dos quatro pedoambientes, referente ao conjunto de parâmetros físicos e químicos na em uma seqüência de transição Campos/Floresta

$\mathrm{Na}$ análise de componentes principais, foram considerados os dois primeiros componentes Fator $1 \mathrm{e}$ Fator 2, pois estes conseguem reter cumulativamente a quantidade suficiente da informação total contida no conjunto das variáveis originais, para cada pedoambiente, que foi definido por 22 variáveis, o que possibilita sua localização com um ponto em um gráfico bidimensional (HAIR et al., 2005).

De maneira geral observou-se que os dois primeiros componentes Fator 1 e Fator 2, armazenaram 74,65\% da variância original dos atributos do solo para os horizontes diagnósticos superficiais. Já os horizontes diagnósticos subsuperficiais apresentaram uma variância acumulada de 73,76\% (FIG. 4). Segundo Sena et al. (2002), para estudos de solos, valores acima de $70 \%$ da variância original acumulada são considerados valores aceitáveis, validando assim as informações do trabalho em questão.
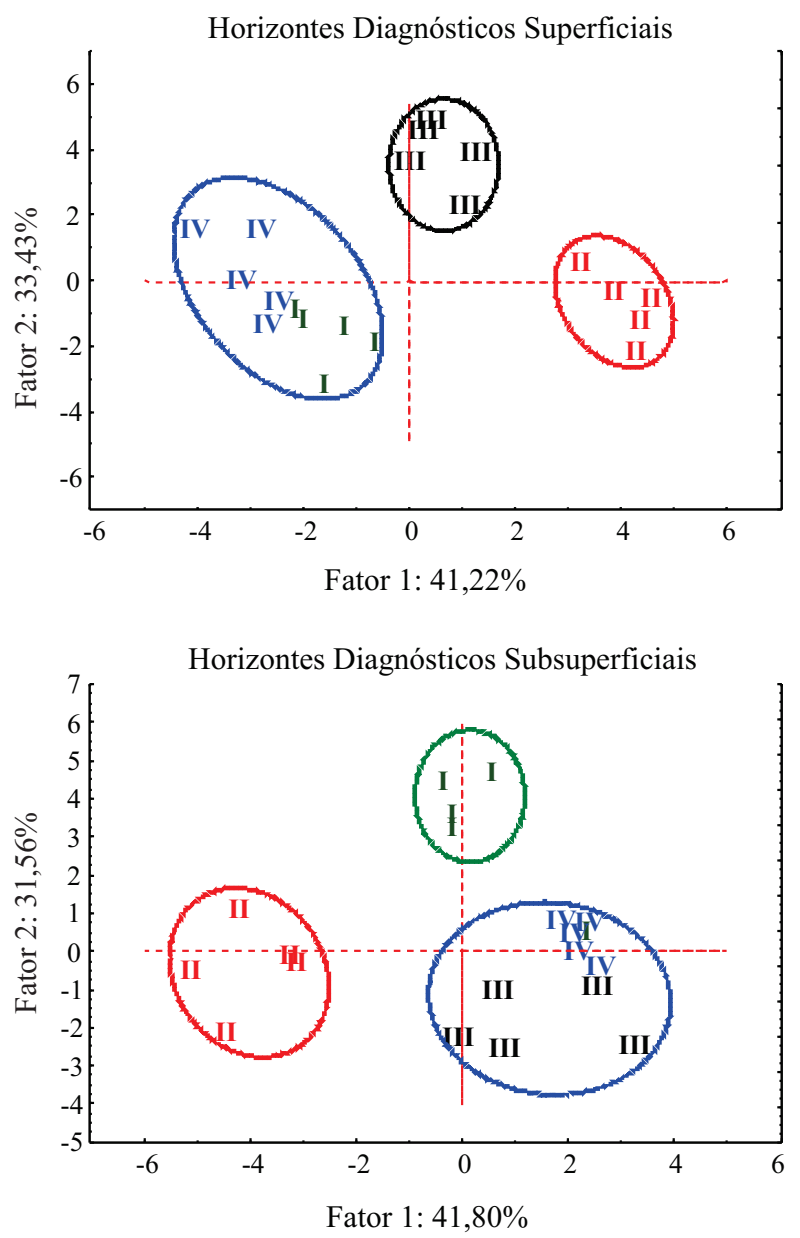

$\mathbf{I}=$ Campo Alto; $\mathbf{I I}=$ Campo Baixo; $\mathbf{I I I}=$ Zona de Ecotóno; $\mathbf{I V}=$ Floresta

Figura 4 - Análise de Componentes Principais para os atributos físicos (areia, silte, argila, S/A, Ds, Dp, Pt) e químicos (pH em água e $\mathrm{KCl}, \Delta \mathrm{pH}, \mathrm{Mg}^{2+}, \mathrm{Ca}^{2+}, \mathrm{K}^{+}, \mathrm{Na}^{+}, \mathrm{Al}^{3+}, \mathrm{H}^{+}+\mathrm{Al}^{3+}, \mathrm{P}, \mathrm{SB}$, $\mathrm{CTC}, \mathrm{V}, \mathrm{m}, \mathrm{MO}$ ) separados por pedoambiente, nos horizontes diagnósticos superficiais e subsuperficiais

A análise de componentes principais (ACP) foi aplicada visando a verificar a relação entre os atributos do solo e os quatro pedoambientes estudados. Na ACP utilizaram-se o conjunto dos atributos físicos e químicos para ambos os horizontes diagnósticos estudados, considerando apenas os dois primeiros eixos fatoriais 
(FIG. 4). Verificou-se que os horizontes diagnósticos superficiais apresentaram, nos dois primeiros eixos, uma variância que explica $74,65 \%$ da variabilidade total dos dados, sendo 41,22 e $33,43 \%$ pelo $1^{\circ}$ e $2^{\circ}$ eixos, respectivamente (FIG. 4). De acordo com Toledo et al. (2009), após esta etapa, a análise multivariada pode ser aplicada na seleção de variáveis relevantes na caracterização e no planejamento de uso sustentável de ambientes naturais.

Em relação aos horizontes diagnósticos subsuperficiais, verificou-se que os dois primeiros eixos apresentaram variância que explica 73,76\% da variabilidade total dos dados, com 41,80 para o primeiro eixo, e $31,56 \%$ para o segundo eixo (FIG. 4).

Com base nos resultados dos horizontes superficiais, constatou-se a formação de três grupos diferentes, um grupo é referente ao conjunto de dados que contemplam os pedoambientes de floresta e campo, outro grupo engloba o pedoambiente de zona de ecótono e um terceiro grupo é formado pelos dados do pedoambiente de campo baixo (FIG. 4). Em relação à análise de componentes principais dos horizontes diagnósticos subsuperficiais, verificou-se a formação de três grupo distintos: um grupo englobando os dados do campo baixo, outro grupo englobou os dados do campo alto e um terceiro grupo agregou os dados da zona de ecótono e floresta. Estes resultados vem confirmar que o uso da análise multivariada de componentes principais pode servir para agrupar indivíduos com características semelhantes e estudar suas correlações (VALLADARES et al., 2008), sendo, neste caso específico, bastante úteis para o estudo dos pedoambientes.

\section{Conclusões}

1. Os atributos físicos e químicos do solo apresentaram-se dependentes dos pedoambientes;

2. A análise de agrupamento permitiu o agrupamento dos solos em três grandes grupos com base nos horizontes superficiais e subsuperficiais, correspondendo a apenas três pedoambientes;

3. A análise de componentes principais para os horizontes diagnósticos superficiais e subsuperficiais mostrouse uma ferramenta útil no estudo das relações entre os atributos do solo e os pedoambientes estudados.

\section{Referências}

BORUVKA, L.; KOZAK, J. Geostatistical investigation of a reclaimed dumpsite soil with emphasis on aluminum. Soil Tillage Research, v. 59, p. 115-126, 2001.
BRASIL. Ministério das Minas e Energia. Projeto Radambrasil, folha SB. 20, Purus. Rio de Janeiro, 1978. 561 p.

BRAUN, E. H. G.; RAMOS, J. R. A. Estudo agroecológico dos campos Puciarí-Humaitá (Estado do Amazonas e Território Federal de Rondônia). Revista Brasileira de Geografia. v. 21, n. 04, p. 443-497, 1959.

CAMPOS, M. C. C. Pedogeomorfologia aplicada a ambientes amazônicos do Médio Rio Madeira. 2009. 242 f. Tese (Doutorado em Ciências do Solo) - Universidade Federal Rural de Pernambuco, Recife.

CARVALHO, A. M. Caracterização física, química e mineralógica dos solos do município de Humaitá-AM. 1986. 166 f. Tese (Livre Docência) Universidade do Estado de São Paulo, Botucatu.

EMPRESA BRASILEIRA DE PESQUISA AGROPECUÁRIA (EMBRAPA). Centro Nacional de Pesquisa de Solos. Manual de métodos de análise de solo. Rio de Janeiro, 1997. 212 p.

EMPRESA BRASILEIRA DE PESQUISA AGROPECUÁRIA (EMBRAPA). Centro Nacional de Pesquisa de Solos. Sistema Brasileiro de Classificação de Solos. Brasília, 2006. 354 p.

FRANZEN, D.W. et al. A survey of soil attributes in North Dakota by landscape position. Agronomy Journal, v. 98, n. 04, p. 1015-1022, 2006.

FREDDI, O. S. et al. Análise multivariada na compactação de um latossolo vermelho cultivado com milho. Revista Brasileira de Ciência do Solo, v. 32, p. 953-961, 2008.

FREITAS, H. A. et al. Florestas X Savanas no passado na Amazônia. Ciência Hoje, v. 32, n. 189, p. 40-46, 2002.

FU, B. J. et al. Relationships between soil characteristics, topography and plant diversity in a heterogeneous deciduous broad-leaved forest near Beijing, China. Plant and Soil, v. 261, n. $01 / 02$, p. $47-54,2004$

HAIR, J. F. et al. Análise multivariada de dados. Porto Alegre: Bookman, 2005.

KAISER, H. F. The varimax criterion for analytic rotation in factor analysis. Psychometrika, v. 23, n. 03, p. 187-200, 1958.

LETEY, J. Relationship between soil physical properties and crop production. Advance in Soil Science, v. 01, p. 277-293, 1985.

MARTINS, A. K. E. et al. Relações solo-geoambiente em áreas de ocorrência de Ipucas na planície do Médio Araguaia - Estado de Tocantins. Revista Árvore, v. 30, p. 297-310, 2006 b.

MARTINS, G. C. Matas e Campos naturais da região de Humaitá (AM): atributos diferenciais dos solos e implicações do uso e manejo. Lavras, 2001.65 f. Dissertação (Mestrado em Solos e Nutrição de Plantas) - Universidade Federal de Lavras.

MARTINS, G. C. et al. Campos nativos e matas adjacentes da região de Humaitá (AM): atributos diferenciais dos solos. Ciência e Agrotecnologia, v. 30, n. 02, p. 221-227, 2006a.

MELO, V. F. et al. Caracterização física, química e mineralógica de solos da colônia agrícola do Apiaú (Roraima, Amazônia), sob 
diferentes usos e após queima. Revista Brasileira de Ciência do Solo, v. 30, n. 06, p. 1039-1050, 2006.

REZAEI, S. A.; GILKES, R. J. The effects of landscape attributes and plant community on soil chemical properties in rangelands. Geoderma, v. 125, n. 01/02, p.167-176, 2005.

ROSOLEN, V.; HERPIN, U. Expansão dos solos hidromórficos e mudanças na paisagem: um estudo de caso na região Sudeste da Amazônia Brasileira. Acta Amazônica, v. 38, n. 03, p. 483-490, 2008.

SANTOS, R. D. et al. Manual de descrição e coleta de solos no campo. 5. ed. Viçosa. Sociedade Brasileira de Ciência do Solo, 2005. 100 p.

SENA, M. M. et al. Discrimination of management effects on soil parameters by using principal component analysis: a multivariate analysis case study. Soil \& Tillage Research, Amsterdam, v. 67, p. 171-181, 2002.

STATISTICA. Statistica 7.0, EUA Software. Tucksa: StatSof, 2005.
TOLEDO, L. O. et al. Análise multivariada de atributos pedológicos e fitossociológicos aplicada na caracterização de ambientes de cerrado no norte de Minas Gerais. Revista Árvore, v. 33, n. 05, p. 957-967, 2009.

VALLADARES, G. S. et al. Análise dos componentes principais e métodos multicritério ordinais no estudo de organossolos e solos afins. Revista Brasileira de Ciência do Solo, v. 32, n. 01, p. 285-296, 2008.

YEMEFACK, M.; ROSSITER, D. G.; NJOMGANG, R. Multi-scale characterization of soil variability within an agricultural landscape mosaic system in southern Cameroon. Geoderma, v. 125, n.01/02, p. 117-143, 2005.

YIMER, F. et al. Soil property variations in relation to topographic aspect and vegetation community in the south-eastern highlands of Ethiopia. Forest Ecology and Management. v. 232, n. 01/03, p. 90-99, 2006.

YOEMANS, J. C.; BREMNER, J. M. A rapid and precise method for routine determination of organic carbon in soil. Communication Soil Science Plant Anal, v. 19, n. 13, p. 1467-1476, 1988. 